\section{Cahiers de Narratologie}

Analyse et théorie narratives

39 | 2021

L'héritage de Ricoeur : du récit à l'expérience

\title{
Diccionario de teorías narrativas 2. Narratología, cine, videojuego, medios
}

\section{Marc Marti}

\section{(2) OpenEdition}

\section{Journals}

Electronic version

URL: https://journals.openedition.org/narratologie/12165

DOI: 10.4000/narratologie.12165

ISSN: 1765-307X

Publisher

LIRCES

\section{Electronic reference}

Marc Marti, "Diccionario de teorías narrativas 2. Narratología, cine, videojuego, medios", Cahiers de Narratologie [Online], 39 | 2021, Online since 29 June 2021, connection on 19 April 2022. URL: http:// journals.openedition.org/narratologie/12165 ; DOI: https://doi.org/10.4000/narratologie.12165

This text was automatically generated on 19 April 2022.

Article L.111-1 du Code de la propriété intellectuelle. 


\title{
Diccionario de teorías narrativas 2. Narratología, cine, videojuego, medios
}

\author{
Marc Marti
}

\section{REFERENCES}

Penguin Random House Grupo Editorial, Caligrama, 2021, 1956 p.

1 Par sa taille et son contenu, l'ouvrage dont nous allons traiter représente une somme colossale de coordination et de travail de synthèse sur la narratologie dans les domaines du cinéma, du jeu vidéo et des médias. Avec presque deux mille pages, c'est sans doute à ce jour l'ensemble le plus complet sur les théories narratives appliquées aux médias audiovisuels et intermédiaux « en langue non anglaise ». L'auteur est un familier des grandes synthèses. Lorenzo Vilches est en effet professeur émérite à l'UAB (Universidad Autónoma de Barcelona) et il a été directeur de nombreuses collections en communication chez les grands éditeurs espagnol et catalans, comme Fontanella, Paidós, ou Gedisa. Il est l'auteur de nombreux ouvrages sur la théorie de l'image, du cinéma, de la photographie, de la télévision et des technologies du numérique. Il a publié un ouvrage en français, La télévision dans la vie quotidienne. État des savoirs, Apogée, 1995. Il a publié comme éditeur aux éditions Gedisa La investigación en comunicación. Métodos y técnicas en la era digital, (2011), ainsi que Convergencia y trasmedialidad. La ficción después de la TDT en Europa e Iberoamérica (2013). Le dernier grand ouvrage en date est Diccionario de teorías narrativas. Cine, televisión transmedia, Caligrama, Penguin Random House, 2017, qui préfigure celui qui nous occupe dans ce compte-rendu.

Le livre est publié en version papier et en ebook, ce qui est une excellente initiative pour un dictionnaire où la recherche par mot-clé est une pratique courante. Sa structuration, malgré l'appellation de dictionnaire, est une hybridation entre un répertoire d'entrées de type glossaire explicatif, un ouvrage collectif avec des articles 
d'une longueur classique (environ vingt-cinq mille signes) et une robuste bibliographie. Cette dernière peut sans nul doute être qualifiée d'exhaustive. En effet, regroupée en fin d'ouvrage, elle comporte deux-cent soixante-dix pages et dépasse les deux-mille entrées en allemand, anglais, espagnol, italien et français. Les auteurs réunis sont au nombre de quarante et un, la majorité étant issus de la recherche hispanophone péninsulaire et latino-américaine, ainsi que cinq participants français, deux brésiliens et un auteur italien.

3 Dans un usage classique, la structure de l'ouvrage offre tous les outils nécessaires à sa bonne utilisation: un index thématique est complété par un index alphabétique. Les entrées renvoient à la définition principale en cas de synonymie (par exemple pour evento et acontecimiento). Certains termes sont aussi regroupés dans un article unique, tout en étant signalés dans les entrées alphabétiques : par exemple amateur et geek sont regroupés dans « usager de médias narratifs » (usuarios de medios narrativos). L'ensemble des notices renvoie à la bibliographie utilisée sous forme abrégée, que l'on pourra retrouver, grâce aux dates et aux noms, au format complet dans la dernière partie du dictionnaire.

4 Le propos de l'ouvrage, qui est en continuité avec le premier tome, est de " penser et d'ordonner les concepts fondamentaux pensés par la narratologie » sur les différentes formes de récits (narrativas en espagnol). L'approche est aussi largement interdisciplinaire, recourant à l'histoire, à la philosophie et à l'ethnographie aux côtés des disciplines plus attendues comme les études filmiques, les games studies et l'esthétique. Elle propose aussi un panorama sur l'évolution théorique, mettant en regard les théories classiques et post-classiques, tout en intégrant les dernières approches des sciences sociales. Par exemple, on peut remarquer que les thématiques de genre, ainsi que celles liées à la représentation des femmes et au féminisne sont largement traitées comme l'indique l'introduction et comme le confirme l'index. La question a en effet pesé de façon majeure sur le renouvellement des théorisations actuelles et surtout, sur les créations récentes. Elle est ainsi absolument incontournable au moment d'analyser, avec une perspective historique, l'ensemble des productions culturelles et leurs mécanismes narratifs, qui sont au cœur de ce dictionnaire.

5 Les entrées offrent à la fois des concepts issus de études classiques sur la littérature, comme ceux de narration pseudo-itérative (Genette), de narrateur principal (Prince) ou des nouveautés issues des études postclassiques, comme la narration polychronique (Herman, Prince, Vilches), tout aussi valable pour la littérature que le cinéma. Certaines montrent l'évolution et la continuité de la réflexion théorique, comme celle consacrée à la métalepse, qui occupe cinq pages et renvoie à la fois à la définition initiale de Figure III (1972) et aux dernières publications de John Pier (2016) en passant par les écrits de Marie-Laure Ryan (2004 et 2005). Les «nouveaux classiques »-ce concept nous semble pertinent- sont aussi très visibles dans les articles et dans la bibliographie, comme « trame et intrigue » qui fait une synthèse de différents travaux de Raphaël Baroni.

6 Les entrées sur le jeu vidéo sont tout aussi remarquables, proposant des synthèses très utiles, puisant dans les travaux de la narratologie et de la ludologie françaises et espagnoles et ceux issus de la tradition anglo-saxonne. Sur ce dernier point, c'est d'ailleurs le trait le plus remarquable de l'ouvrage, l'utilisateur (on hésite à parler de simple lecteur pour un dictionnaire) a toujours l'impression de se déplacer dans une immense Babel théorique, parfaitement maîtrisée par l'éditeur, qui prend soin de mettre côte-à-côte les concepts qui semblent exprimés par des termes différents en 
fonction de la langue utilisée et en proposant aussi en espagnol une traduction systématique de l'anglais ou du français. En l'absence d'une œuvre d'une telle ambition dans l'espace francophone, la première chose à souhaiter serait d'ailleurs sans doute une traduction, qui serait d'une grande utilité à la narratologie francophone, déjà traitée de façon exhaustive dans cette version en espagnol.

7 Pour conclure, il s'agit donc, on l'aura compris, d'un ouvrage absolument indispensable pour tout chercheur en narratologie, et ce, quel que soit son domaine. L'outil permettra tout à la fois de lever un doute terminologique, de constituer une synthèse ou de partir de pistes bibliographiques solides sur tout sujet narratologique. 